\title{
Exploring the Pathogenesis, Clinical Characteristics and Therapeutic Regimens of Listeria monocytogenes
}

\section{Kee-Yan Loo ${ }^{1 \mathrm{a}}$, Vengadesh Letchumanan ${ }^{1 \mathrm{a} *}$, Amreeta Dhanoa ${ }^{1}$, Jodi Woan-Fei Law ${ }^{1,2}$, Priyia Pusparajah ${ }^{1,3}$, Bey-Hing Goh ${ }^{4}$, Hooi-Leng Ser ${ }^{1,2}$, Sunny Hei Wong ${ }^{5}$, Nurul-Syakima Ab Mutalib ${ }^{6}$, Kok-Gan Chan $^{7,8 *}$ and Learn-Han Lee ${ }^{1,3 *}$}

${ }^{1}$ Novel Bacteria and Drug Discovery Research Group (NBDD), Microbiome and Bioresource Research Strength (MBRS), Jeffrey Cheah School of Medicine and Health Sciences, Monash University Malaysia, Bandar Sunway, Selangor Darul Ehsan, Malaysia

${ }^{2}$ Institute of Biomedical and Pharmaceutical Sciences, Guangdong University of Technology, Guangzhou, PR China

${ }^{3}$ Medical Health and Translational Research Group (MHTR), Jeffrey Cheah School of Medicine and Health Sciences, Monash University Malaysia, Selangor Darul Ehsan, Malaysia

${ }^{4}$ Biofunctional Molecule Exploratory Research Group (BMEX), Biomedicine Research Advancement Centre (BRAC), School of Pharmacy, Monash University Malaysia, Bandar Sunway, Selangor Darul Ehsan, Malaysia

${ }^{5}$ Li Ka Shing Institute of Health Sciences, Department of Medicine and Therapeutics, The Chinese University of Hong Kong, Shatin, Hong Kong ${ }^{6}$ UKM Medical Molecular Biology Institute (UMBI), UKM Medical Centre, University Kebangsaan Malaysia, Kuala Lumpur, Malaysia ${ }^{7}$ Division of Genetics and Molecular Biology, Institute of Biological Sciences, Faculty of Science, University of Malaya, Kuala Lumpur, Malaysia ${ }^{8}$ International Genome Centre, Jiangsu University, Zhenjiang, PR China

${ }^{a}$ These authors contributed equally to this writing

*Corresponding Author: Learn-Han Lee, Jeffrey Cheah School of Medicine and Health Sciences, Monash University Malaysia, Selangor, Malaysia.

E-mail: lee.learn.han@monash.edu; leelearnhan@yahoo.com; Kok-Gan Chan, Institute of Biological Sciences, Faculty of Science, University of Malaya, Kuala Lumpur, Malaysia. E-mail: kokgan@um.edu.my; Vengadesh Letchumanan, Jeffrey Cheah School of Medicine and Health Sciences, Monash University Malaysia, Selangor, Malaysia. E-mail: vengadesh.letchumanan1@monash.edu

DOI: 10.31080/ASMI.2020.03.0531
Received: February 04, 2020

Published: February 20, 2020

(C) All rights are reserved by Kee-Yan Loo., et al.

\begin{abstract}
Listeria monocytogenes is a ubiquitous Gram-positive bacterium widely distributed in the soil where it lives as a saprophyte. This opportunistic pathogen causes listeriosis in humans through consumption of contaminated food especially ready-to-eat (RTE) food, chiefly deli meat, cheese, and smoked fish. Pregnant women, neonates, the elderly, and immunocompromised patients are especially vulnerable to listeriosis. The pathogenesis of listeriosis begins with ingestion of the bacterium by humans; low $\mathrm{pH}$ and increased gastric temperature in the gastrointestinal system upregulates the production of virulence proteins, for example PrfA and internalins, facilitating the transition of L. monocytogenes from its saprophytic existence to a pathogenic one. Internalins mediate its adherence and invasion of host intestinal epithelial cells which are usually non-phagocytic. Inside the phagosome, the low carbohydrate and low iron concentration suppress internalin production. The production of listeriolysin O (LLO) and PlcA then allows the lysis of the phagocytic vacuole, enabling the entry of bacteria into the cytosol. High levels of ActA and PlcB expression promotes cell-to-cell spread. Understanding the pathogenesis of L. monocytogenes can help appreciate the microbial virulence, treatment options, and the development of better therapeutic agents for infected patients. Therefore, this review focuses on the pathogenesis $L$. monocytogenes, as well as the clinical characteristics, and therapeutic regimens that would be beneficial in the management of the disease.
\end{abstract}

Keywords: Listeria monocytogenes; Gram-Positive; Listeriosis; Contaminated Food; Internalins 


\section{Abbreviations}

L. Monocytogenes: Listeria monocytogenes; RTE: Ready-To-Eat; EU: European Union; WHO: World Health Organization; BSH: Bile Salt Hydrolase; PRFA: Positive Regulatory Factor; GIT: Gastrointestinal Tract; LRR: Leucine Rich Repeat; PLC: Phospholipase; LLO: Listeriolysin; AGR: Accessory Gene Regulatory; CNS: Central Nervous System; TMP-SMX: Trimethoprim-Sulfamethoxazole

\section{Introduction}

Foodborne diseases are a growing public health problem that effects all age group of people globally. The World Health Organization (WHO) has estimated nearly 600 million people fall ill and 420,000 die due to foodborne diseases annually; this impedes a country's socioeconomic development by straining the healthcare system, economy, tourism, and trade [1]. The top three foodborne pathogens identified by the WHO are Salmonella sp., Listeria sp., and Vibrio sp. which are responsible for many foodborne associ- ated outbreaks worldwide. Unlike Salmonella sp. and Vibrio sp., Listeria sp. is able to survive in stressful conditions, therefore understanding the pathogenesis of Listeria monocytogenes may lead to better disease management and treatment advances.

Listeriosis is caused by Listeria monocytogenes, the only member in the genus Listeria that is a human pathogen. This facultative intracellular Gram-positive bacillus has been reported as a causative agent of foodborne infection in humans and animals since the 1970s [2]. Listeria monocytogenes has the ability to resist environmental stresses, for example, low $\mathrm{pH}$ levels or high sodium chloride (salt) concentration, which makes the bacterium a concern for the food production sectors [3]. It is often isolated from soil, water, food products, ready-to-eat food, animal and human carriers [4]. The clinical manifestation of listeriosis can be severe with prolonged hospitalization and high mortality rates. Figure 1 diagrammatically shows the Listeria monocytogenes infection to humans.

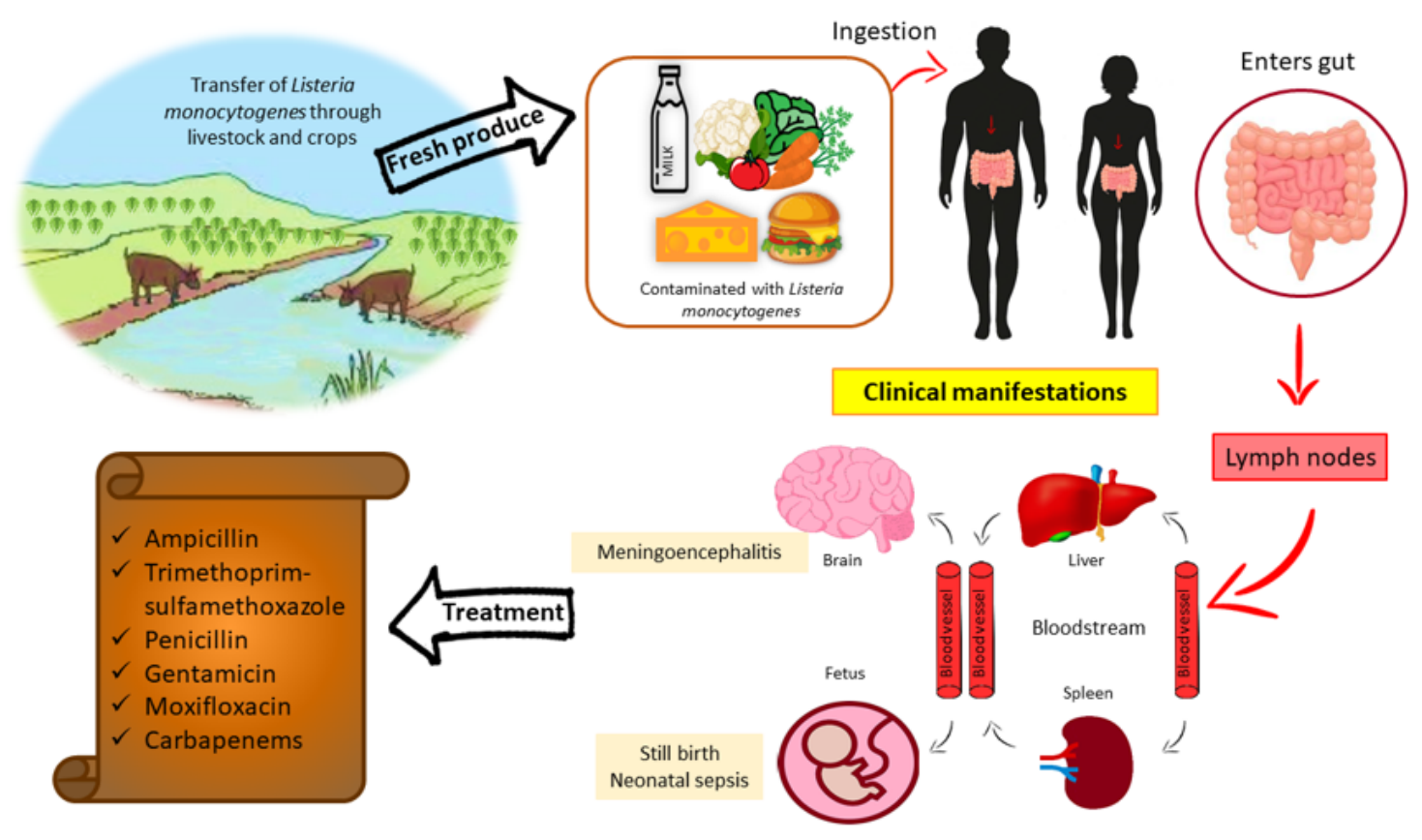

Figure 1: Illustration of Listeria monocytogenes infection. Listeria monocytogenes that is found in soil and water is transferred through livestock and crops to humans. Upon ingestion of contaminated food, Listeria monocytogenes traverse the intestinal barrier and spread to the bloodstream through the lymph nodes. It disseminate in the liver and spleen of the host. Listeria monocytogenes has the ability to cross the blood-brain barrier and fetoplacental causing meningoenceohalitis barrier and still birth or neonatal sepsis respectively. Immunosuppressed patients and pregnant mothers are in higher risk and present severe symptoms. Listeria monocytogenes infections can be treated with antibiotics including ampicillin, trimethoprim-sulfamethoxazole, penicillin, gentamicin, moxifloxacin and carbpenems.

Since the 1990s, the prevalence of L. monocytogenes in various food has been reduced with adequate control measures taken by the regulatory bodies [5]; however, the rate of disease has continued to increase over the last few decades. The European Union (EU) have continuously reported outbreaks and sporadic cases of listeriosis. In 2011, the (EU) reported approximately 1470 cases of human infection with $12.7 \%$ mortality rate [6]. The figure increased to 1763 confirmed human listeriosis cases in 2013 with
$99.1 \%$ requiring hospitalization and $69.7 \%$ mortality rate [7]. The majority of the cases were domestically acquired through food such as crustaceans, shellfish, cheese, dairy products, meat and vegetables [7]. The United States have reported frequent listeriosis outbreaks attributed to various food including pre-cut diced celery served in a Texas hospital resulting in 5 deaths [8], ice-cream [9], cantaloupe in Colorado and several states [10], mung bean sprouts in Illinois and Michigan [11], and pre-packed caramel apples in 
several states with 7 fatalities [12]. All these confirmed cases affected patients from all age groups including pregnant women and newborn infants. The largest ever documented listeriosis outbreak happened in South Africa [13]. The outbreak was serious with high fatality rates affecting neonates, the elderly, and immunocompromised patients [5]. A total of 1034 confirmed listeriosis cases -including 400 neonatal cases and in total resulting in 204 deaths were reported from January, 2017 to May, 2018 [13]. The source of the majority of the listeriosis was reported to be "polony", a South African type of deli meat made of pork [14]. The Department of Health South Africa and WHO investigated the cause of listeriosis, recalled all the products and banned the export of polony from South Africa in March 2018 [15].

Listeria monocytogenes adapts well in the human gastrointestinal system and is able to overcome the acidity changes, osmolarity, oxygen differences, and effects of antimicrobial peptides and bile [16]. Bile salts actually act as a trigger for several bacteria to initiate their virulence genes allowing them to cause infections in the human gastrointestinal system [17]. However, this is not the case for L. monocytogenes, which has a specific putative gene encoding a bile salt hydrolase (BSH). Recently, a study established BSH as a novel PrfA-regulated L. monocytogenes virulence factor that helps with its invasion during the intestinal and hepatic phases of listeriosis [18]. These findings provide an insight into the pathogenicity of this pathogen in the human system during the course of infection. Since L. monocytogenes thrives well under stressful environmental conditions, it will be difficult to eradicate it without carefully studying its pathogenesis and virulence factors. Hence, this review is aimed at discussing the pathogenesis of L. monocytogenes as well as the clinical characteristics and therapeutic regimens that would be beneficial in the management of the disease.

\section{Pathogenesis of Listeria monocytogenes}

Listeria monocytogenes has three distinct life-styles: 1) intracellular - an actin-based motility for cytoplasmic movement and cellto-cell spread, 2) extracellular - a free-living, flagellum-propelled bacterium in the environment, and 3) extracellular - a member of biofilm communities [19]. Even during its peaceful, saprophytic existence, $L$. monocytogenes has the ability to utilize a variety of carbon sources to produce an arsenal of gene products which ensure its survival in its natural environment [20]. It becomes pathogenic when it undergoes transition from its saprophytic life through altered expression of various complex regulatory pathways [21]. These virulence genes and perfectly regulated pathways have been of great scientific interest, as they may contain potential targets of inhibition to control the life cycle of L. monocytogenes. Prior studies have used guinea pigs and transgenic mice expressing human Ecadherin in enterocytes to study the virulence of L. monocytogenes [22]. Recent discoveries identified that the gut microbiota played an important role during the orally acquired $L$. monocytogenes infections. The host gut microbiota interferes with microRNA (miRNA) expression upon oral infections [23]. This interference reduces $L$. monocytogenes colonization of the gut and systemic dissemination [24]. It is identified that treatment with Lactobacillus decreases the invasion abilities of L. monocytogenes in the host [25].

Listeria monocytogenes shift through a series of different morphological stages once it enters into the gastrointestinal tract [26] (Figure 2). It will first adhere to the enterocytes and induce its entry the cell through endocytosis. Upon internalization, the phagosome will lyse and the actin filaments are reorganized from a cloud into a tail (also known as comet tail) to allow migration within the cytoplasm and form an association with the cell membrane.

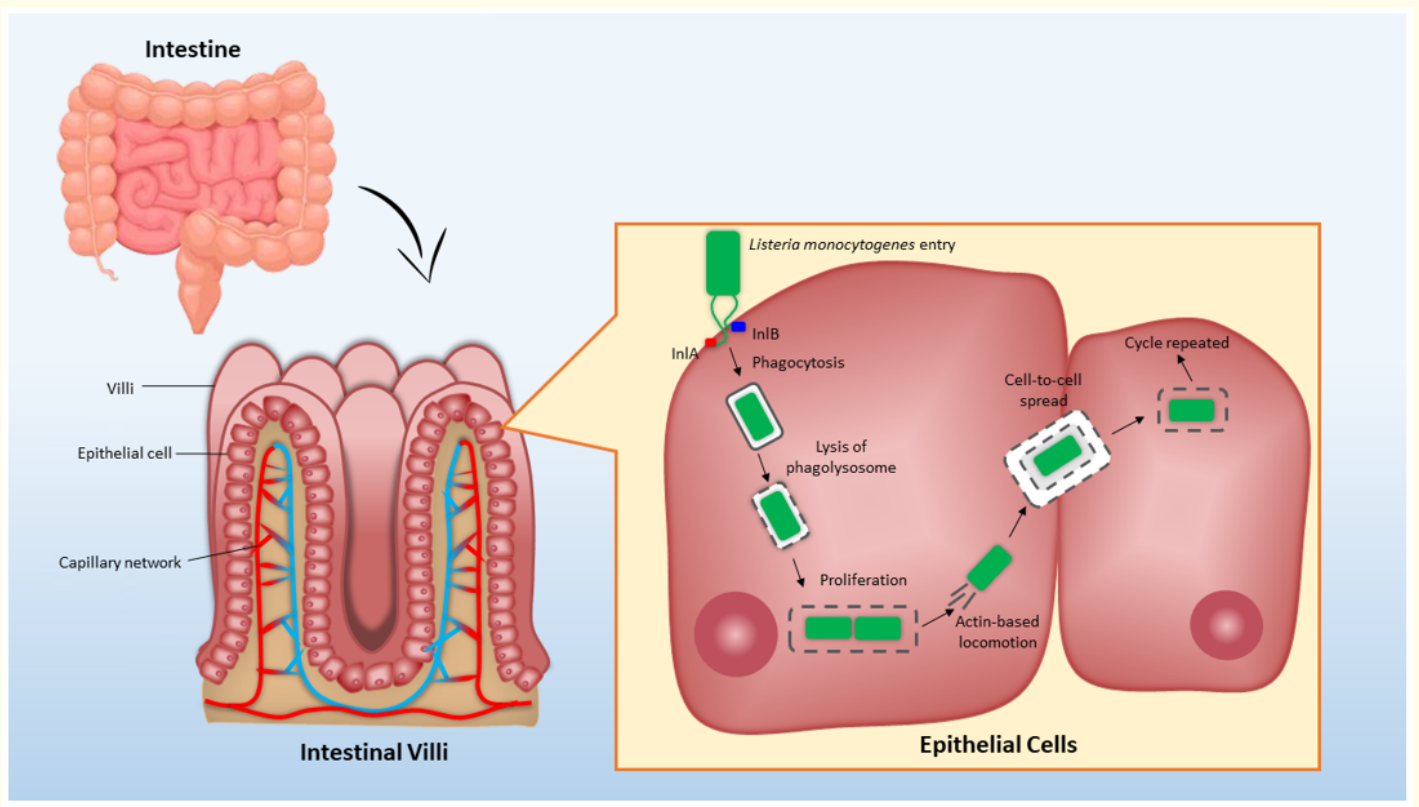

Figure 2: Pathogenesis of Listeria monocytogenes in host. Listeria monocytogenes enters into the host and requires interactions of surface internalins InIA and InIB with their respectice cell surface receptors E-cadherin and Met. The endocytic vacuole rupture via Listeriolysin $\mathrm{O}$ (LLO), phospholipase A (PlcA) and phospholipase (PlcB). Listeria monocytogenes is able to replicate in the cytosol by utilizing the cytosolic resources to their own benefit. The bacterial surface protein ActA stimulates the polymerisation of cellular actin. The actin filaments polarize at one of the bacteria, which allow intracellular motility, and it spread from cell-to-cell. 
Recognition of bacteria-containing pseudopods by neighboring cells allows internalization of the pseudopod into the associated cell, resulting in a double-membrane phagosome. The both membrane solubilize and releases the bacteria into the cytoplasm. It is important to highlight that this type of spreading enables the pathogen to evade host immunity surveillance, thus cell-mediated immunity is crucial to eliminate the microbe [26]. Thus, conditions causing suppression of cell-mediated immunity can predispose to listeriosis. In order to understand how L. monocytogenes actually becomes such a successful pathogen, it is of great importance to first discuss the "mastermind" genes that are responsible for the expression of its virulence factors.

\section{Regulators for gene expression}

\section{Positive regulator factor (PrfA)}

$\operatorname{PrfA}$ is a $27 \mathrm{kDa}$ transcriptional activator protein that regulates the expression of virulence genes: $p r f A, p l c A, p l c B$, inl $A$, inlB, inlC, $h l y, m p l$, vip, hpt, and bsh [27]. These genes regulated by $\operatorname{PrfA}$ and have a binding site (PrfA box) which consists of a 14 bp palindromic sequence in the -41 region. The differential expression of $\operatorname{PrfA}$ dependent genes is affected by variations of PrfA box sequences, and whether their promoters can be efficiently transcribed at low $\operatorname{PrfA}$ levels [20].

The $p r f A$ is located on the $p l c A-p r f A$ operon, which can self-regulate its expression (autoregulation). The plcA promoter (PplcA) is located upstream of $p l c A$ and is responsible for the transcription of monocistronic plcA and bicistronic plcA and prfA. Two prfA promoter regions, $\operatorname{PprfA_{1}}\left(\sigma_{\mathrm{A}}\right.$-dependent) and $\operatorname{PprfA_{2}}\left(\sigma_{\mathrm{A}^{-}}\right.$and $\sigma_{\mathrm{B}}{ }^{-}$ dependent) have been identified at the immediate upstream of prfA coding region and both are responsible for directing the transcription of monocistronic prfA $[20,27,28]$. Mutant strains with a deletion of either $\operatorname{Pprf}_{A 1}$ or $\operatorname{Pprf}_{A 2}$ demonstrated full virulence, indicating the functional redundancy of these two promoters [20].

The PprfA, RNA transcript contains an mRNA sequence located in the 5' untranslated regions (5' UTRs) acting as a thermo-sensor riboswitch that is able to perform cis-regulation. This riboswitch melts at high temperature, thus allowing the translation of $\operatorname{prfA}$, and forms a stable stem-loop structure that masks the ribosome binding region at temperatures $<30^{\circ} \mathrm{C}$, thus inhibiting the translation. The $\mathrm{PprfA} A_{2}$ transcript, by contrast, does not contain a thermosensitive structure, thus its translation may account for the expression of $\operatorname{prf} A$ in some low temperatures such as outside the mammalian host cells [20, 29]. Recently, two S-adenosylmethionine (SAM)-binding riboswitches, SreA and $\operatorname{SreB}$, have been shown to exert modest regulatory effects the expression of PrfA post transcriptionally, but their biological significance and detailed pathways currently remain unclear [29].

The transition of a saprophytic life-form to a pathogenic one within a host is postulated to be triggered by the availability of specific types of nutrients. In the presence of carbohydrates usually transported through the phosphoenol pyruvate sugar phosphotransferase system, such as extracellular cellobiose, $\operatorname{PrfA}$ activity is strongly inhibited; however $\operatorname{PrfA}$ activity appears to be induced in the presence of intracellular hexose derivatives. This appears to be via activation rather than synthesis as levels of $\operatorname{PrfA}$ remain constant $[20,29,30]$. Given that $\operatorname{PrfA}$ is a member of the cyclic AMP receptor protein-fumarate and nitrate reduction regulator ( Crp/Fnr) family, it requires binding with small molecule cofactors for complete activation; however the identity of these molecules is currently unknown $[29,30]$. A recent study of electrostatic modeling of PrfA revealed three putative cofactor binding regions: K64, K122, and K130. A substitution in either K62 or K122 induced defective intracellular actA expression; conversely, double substitution of K64/K122 restored a portion of PrfA activity, suggesting that charge neutralization mimics the condition of cofactor binding. Substitution of K130 resulted in a conformational change that completely abolished the activation of PrfA. A mutant G145S induced a conformational change that is dominant over any alteration in the cofactor binding region whereby PrfA is active even in the absence of co factor binding [29]. Nevertheless, the genetic basis of chemotaxis towards nutrient sources as yet remains elusive as the $\operatorname{PrfA}^{*} \mathrm{G} 145 \mathrm{~S}$ mutant demonstrated severely compromised flagellum-mediated swimming motility despite enhanced virulence [29].

\section{Sigma factors}

Five major sigma factors have been identified in L. monocytogenes: $\sigma^{\mathrm{A}}, \sigma^{\mathrm{B}}, \sigma^{\mathrm{C}}, \sigma^{\mathrm{H}}$ and $\sigma^{\mathrm{L}}$. The primary sigma factor is $\sigma^{\mathrm{A}}$ and it influences the specificity of RNA polymerase on the $\operatorname{Pprf}_{A 1}$ mRNA transcript in actively growing, unstressed cells [20]. Recently discovered $\sigma^{\mathrm{C}}, \sigma^{\mathrm{H}}$, and $\sigma^{\mathrm{L}}$, known as the alternative sigma factors, are suggested to fine-tune gene expression and protein production under different environmental conditions. Further details about their exact roles in PEP-phosphotransferase systems (PTS) needs to be explored [31].

The most studied sigma factor is $\sigma^{\mathrm{B}}$, also known as general stress responsive sigma factor, positively regulates at least 160 genes [32]. It is crucial for environmental adaptation such as during carbon starvation, low $\mathrm{pH}$, extreme temperatures, oxidative stress, and salty environment $[32,33]$. The $\operatorname{Pprf} A_{2}$ is partially influenced by $\sigma^{\mathrm{B}}$ because the level of PrfA was not significantly reduced in $\Delta$ sigB [28]. In other words, this indicates that prfA expression is not predominantly dependent on the $\sigma^{\mathrm{B}}$-regulated $\mathrm{Pprf} A_{2}$ promoter. However, $\sigma^{\mathrm{B}}$ plays a pivotal role during gastrointestinal epithelial cell invasion due to its role in activating the promoter of inlA $\left(\mathrm{P} 4_{\text {inla }}\right)$ and putative promoter of inlB $\left(\mathrm{P} 2_{\text {inlB }}\right)$. The $\sigma^{\mathrm{B}}$ factor does not contribute to systemic spread of $L$. monocytogenes because the virulence of $\Delta \operatorname{sig} B$ was not attenuated in the intravenously infected guinea pigs $[19,28]$. A few studies have been recently published about the discovery of the role of $\sigma^{\mathrm{B}}$ in antimicrobial resistance as $\sigma^{\mathrm{B}}$ caused resistance to bacterial cell wall-acting antibiotics, for example, nisin, lacticin 3147, ampicillin, penicillin $\mathrm{G}$, and vancomycin [34]. PrfA, sigma factors and heat shock related negative regulators (CtsR and HrcA) are among the gene expression regulators that have been discovered, but only PrfA and $\sigma^{\mathrm{B}}$ are well-studied. Interestingly, there appears to be significant overlapping of these regulator genes with $\sigma^{\mathrm{B}}$ playing the main role of co-regulating the expression of other regulator genes, suggesting the complex, synergistic, and compensatory role of the regulators [35]. 


\section{Other virulence regulator genes}

The L. monocytogenes virulence regulation depends on the PrfA, which controls the expression of genes and major virulence factors. In spite of this, there are several other important regulators for examples VirR [36], Hfq [37], MogR [38], GmaR [39], and MouR [40] contributing to a lesser extent in the virulence regulatory network of L. monocytogenes. VirR is a response regulator component that was discovered in a transposon mutagenesis screen to identify $L$. monocytogenes mutants that exhibited lesser virulence in an infected mouse model [41]. In this study, Madin and colleagues also discovered VirS as the cognate sensor kinase for VirR. In general, the VirR have revealed to control the transcription genes that are involved in the defense against cell envelope stress.

Another regulator gene, $\mathrm{Hfq}$ is an important regulator factor of physiological processes that include stress resistance and virulence. This RNA-binding protein is involved in L. monocyctogenes to tolerate osmotic and ethanol stress, as well as ensure a long term survival in a limited conditions [37]. Recently, Pinheiro and colleagues identified MouR involved in the L. monocytogenes virulence regulation [40]. The study revealed that the MouR is the first regulator of the Agr system, biofilm formation and the host immune response, thus promoting L. monocyctogenes virulence.

Adhesion and internalization into mammalian cells

To establish an infection, L. monocytogenes first has to overcome various gastrointestinal tract (GIT) defense mechanisms, for example, gastric secretion, peristalsis, and the mucus layer lining of the GIT. It needs to adhere to the intestinal epithelial cells and get internalized into the cells, so that it can be shielded from host humoral immunity. Strains that adhere strongly to mammalian cells result in a more severe pathology than weakly adherent strains $[42,43]$. Internalins are surface proteins on L. monocytogenes that play a vital role in invading host cells via cadherin transmembrane proteins and Met receptors. L. monocytogenes internalins are comprised of InlA, InlB, InlC, InlC2, InlD, InlE, InlF, InlG, InlH, InlI, InlJ, and InlK. However, only InlA and InlB are well-studied and known to play vital roles in the invasion of host cells, whereas the putative virulence role of the others remains unclear $[44,45]$. Both these surface proteins have different attachment sites; the InIA binds to E-cadherin receptor, whereas InIB binds to hepatocyte growth factor receptor (Table 1) [46]. All internalins consist of a signaling region, leucine rich repeat (LRR) domain, conserved inter-repeat (IR) region, and LPXTG domain, in which the latter is associated with cell wall adhesion by sortases. The exception to this is InlB, which has $3 \mathrm{GW}$ peptides as anchoring motif, rather than LPXTG $[42,45]$.

The InlA gene is an 800-amino acid protein containing 15 LRRs and IR, which appears to specifically depend on the proline at position 16 (Pro16) of E-cadherin for recognition [47]. E-cadherin is a polypeptide of 882 amino acids that is found in the intestinal epithelial cells. The surface protein InlA's receptor on intestinal

\begin{tabular}{|c|c|}
\hline Actions & Proteins/Function \\
\hline Regulation & $\begin{array}{l}\text { Positive regulatory factor A - PrfA } \\
\text { It functions as a central virulence regulator } \\
\text { of virulence gene transcription. } \\
\text { SigmaB } \\
\text { It is a stress transcription factor }\end{array}$ \\
\hline $\begin{array}{l}\text { Attachment and } \\
\text { invasion }\end{array}$ & $\begin{array}{l}\text { Internalin A - InIA } \\
\text { It is the main internalin responsible for at- } \\
\text { tachment and invasion. InIA and E-caderin } \\
\text { is vital for translocation of L. monocytogenes. } \\
\text { Internalin B - InIB } \\
\text { InIB protein function to bind with hepato- } \\
\text { cyte growth factor receptor. } \\
\text { Internalin F - InIF } \\
\text { A surface protein with specific relevance for } \\
\text { infection of host brain. } \\
\text { Listeria adhesion protein - LAP } \\
\text { Promotes the translocation of Listeria mono- } \\
\text { cytogenes across the intestinal barrier. }\end{array}$ \\
\hline Lysis of vacuoles & $\begin{array}{l}\text { Listeriolysin O - LLO } \\
\text { It enables Listeria monocytogenes to escape } \\
\text { into the cytosol upon internalization. } \\
\text { PC-PLC } \\
\text { It is encoded by plcB and has a role of hydro- } \\
\text { lyzing a wide range of phospholipids }\end{array}$ \\
\hline $\begin{array}{l}\text { Cell-to cell } \\
\text { spread }\end{array}$ & $\begin{array}{l}\text { ActA } \\
\text { It is an actin assembly inducing protein } \\
\text { involved in cell to cell spread. } \\
\text { Listeriolysin } \mathbf{0} \text { - LLO } \\
\text { It has the ability to damage the plasma } \\
\text { membrane of host cells. }\end{array}$ \\
\hline
\end{tabular}

Table 1: Stress response and virulence associated proteins of Listeria monocytogenes.

epithelial cells is E-cadherin, thus making it vital for translocation of L. monocytogenes across the intestinal barrier [48,49]. L. monocytogenes has the ability to invade the tip of intestinal villi at the site of invasion [50]. Nikitas and colleagues demonstrated this ability and affirmed that this bacterium is transcytosed across the intestinal epithelium [48]. Besides serving as a receptor for InlA, the ligand and receptor engagement also plays a role in internalization by promoting actin polymerization [45]. The InlA has a pivotal role in the early stage of GIT infection but none during invasive infection or systemic spread. Recently, its role in placental invasion was confirmed; however, both InlA and InlB are required to recognize their respective receptors, E-cadherin and Met, in order to cross the placental barrier $[23,42,45]$. Evidence has shown that L. monocytogenes strains, specifically ScottA strain are associated with pregnancy related cases causing stillbirth and maternofetal infections [51]. 
Likewise, InlB, is a 600 -amino acid protein comprising seven LRRs, with its gene located immediately downstream of inlA. It mediates the entry of L. monocytogenes into broad cell lines, including hepatocytes and non-epithelial cells by recognizing hepatocyte growth factor receptor (Met), gC1qR, and heparin sulfate proteoglycan $[27,44,52]$. Research has shown that the $\Delta$ inlB strain reduced the invasion capacity more for HepG-2 cells than Caco-2 cells; whether the $\triangle i n l B$ strain has less severe invasion defect compared with $\triangle i n l A$ strain remains to be established due to contradictory results [28].

The exact roles of internalins InlC, InlI, InlJ, and InlH have not been well established. Different from other internalins, InlC does not have an anchoring motif and is secreted extracellularly. Recent studies proposed its role in supporting the function of InlA [20,44]. The largest internalin is InlI; however, its function is unknown [22]. InlJ is an 851-amino acid protein comprising 15 LRRs, and its LRRs consensus is only made up of 21 residues [22]. This newly discovered internalin is absent from non-pathogenic Listeria species. Its expression is temperature dependent but surprisingly, not regulated by major listerial regulators, suggesting its regulators have yet to be identified. An increased transcription level was detected when temperature increased from $16^{\circ} \mathrm{C}$ to $30^{\circ} \mathrm{C}$; however there was no change in the level of InlJ protein production, suggesting post-transcriptional control [21]. A novel internalin, InlK, made up of 578 residues, has a classic LRR domain followed by three distinct folding domains (known as "bent arm"). This "bent arm" is highly flexible, allowing repositioning to accommodate the other partner molecule as needed. The InIK is the first internalin known to participate in L. monocytogenes escape from autophagy by recruiting major vault protein (MVP) in host cells. However, InlK and MVP interaction still remains a subject of great interest [52]. Recently, a study reported InIF is important to L. monocytogenes in order to achieve optimal colonization in murine brain. This suggests that InIF is bacterial surface protein with specific relevance for facilitating infection of the host's brain, thus increasing the pathogenicity of the bacteria [36].

It is important to highlight that among the internalins, the invasion of human non-professional phagocytic cells is primarily mediated by InlA and InlB, and their expression can be regulated by either PrfA or $\sigma^{B}$. To establish listeriosis through an oral route, InlA, but not InlB, plays a critical role in crossing the intestinal barrier. A recent study demonstrates that InlB, InlC, and InlJ bind to major components of intestinal mucus (MUC2) but their interaction needs to be further investigated, as well as the role of other internalins in the pathogenesis of listeriosis [53]. Other proteins such as $\mathrm{P} 60$, fibronectin binding protein ( $\mathrm{FbpA})$, Auto, and Vip are suggested to have a role in mediating L. monocytogenes entry into the host cell. A recent study has demonstrated the role of FbpA in liver and intestinal colonization $[27,43]$. In addition, L. monocytogenes utilizes Listeria adhesion protein (LAP) to exploit epithelial defenses and cross the intestinal epithelial barriers [54,55]. The LAP is a protein that promotes adhesion of pathogenic Listeria species to intestinal cells [56]. The insulin-like growth factor II receptor (IGFIIR), also known as cation-independent mannose6-phosphate receptor (M6PR), was also identified to be possibly involved in attachment and internalization [57]. Since not many studies have been done to describe their mechanisms, they cannot as yet be included among the dominant players in pathogenesis of listeriosis.

\section{Escape from phagosome and intracellular growth}

After successful entry into the host cells, listeriolysin (LLO) in concert with 2 main phospholipases aid L. monocytogenes to escape from its phagosome, without triggering host immune surveillance. Within host cytosol, the bacteria utilize host nutrients to support growth and continue their intracellular life cycle.

\section{Listeriolysin 0 (LLO)}

Listeriolysis O (LLO) is a cholesterol-dependent pore-forming cytolysin, encoded by $h l y$, which enables the bacteria to escape into the cytosol upon internalization. However, its exact mechanism of phagosome destruction and bacteria escape is not well-documented $[20,27]$. Unlike other thiol-activated toxins, it is maximally activated at $\mathrm{pH} 5$ and inactivated at $\mathrm{pH} 7$, thus impairing its devastating effect on plasma membrane when L. monocytogenes is free in cytosol [27]. Recently, LLO was suggested to induce autophagy, which is an innate immune defense against intracellular pathogen, to limit replication of bacteria. However, it does not show any bacterial growth effect, thus postulating its role in enhancing bacteria to escape rather than regulating replication [58]. Listeriolysin O (LLO) is capable of forming spacious Listeria-containing phagosomes (SLAPs) in immunocompromised mice by blocking acidification across the SLAP membrane, thus preventing fusion of phagosome with lysosome and allowing the bacteria to continue growing within the vacuole. The host is able to sustain bacteria viability within the cytosol but unable to eradicate it [59].

\section{Phospholipase (PLC)}

Listeria monocytogenes secretes two types of phospholipases: phosphoinositide-phospholipase C (PI-PLC) and broad-range phospholipase C (PC-PLC). Both work in synergy with LLO to allow bacteria to escape from vacuole. The PI-PLC is encoded by plcA. It catalyzes the breakdown of membrane phospholipid into diacylglycerol (DAG) and inositol phosphate, and subsequently activates the host calcium-dependent protein kinase C (PKC) cascade. The resultant activation of PKC $\beta$ isoforms permeabilizes the vacuole membrane and allows bacteria to escape from the macrophages phagosome but this mechanism does not induce escape within human epithelial cells [60]. It is important to highlight that both PIPLC and LLO work together in permeabilizing the primary vacuole because PI-PLC is believed to enter the host cell cytosol via pores formed by LLO [27].

The PC-PLC is encoded by $p l c B$ and has a role of hydrolyzing a wide range of phospholipids, including phosphatidylcholine, phosphatidylserine, phosphatidylethanolamine, and sphingomyelin 
$[20,27]$. It is synthesized as a 24 amino acid proenzyme into the host cell cytosol and later accumulates at the membrane-cell wall interface. A drop in $\mathrm{pH}$ during cell-to-cell spread results in a rapid translocation of the proenzyme, which is associated with metalloprotease-dependent cleavage to form mature PC-PLC. Unlike PIPLC, it can act in the absence of LLO to promote lysis of primary phagosome in human epithelial cells. It is also involved in efficient cell-to-cell spread and the lysis of secondary vacuoles after the spread. However, the details of these mechanisms have not been well-studied [20,27].

\section{Intracytoplasmic movement and cell-to-cell spread}

Within the host cell, movement of L. monocytogenes is dependent on the assembly of actin. Typically, 3 to 5 hours post infection, intercellular spread begins [58]. The bacteria are spread by the formation of pseudopods in the host cell, which are later internalized into the neighboring cell. It has been suggested that bacteria are released from the protrusion phase, a similar mechanism seen in Streptolysin O from Group A Streptococcus [61].

\section{ActA}

The ActA is a 639-amino acid, actin polymerization or recruiting protein comprised of a signaling amino terminal domain and a transmembrane carboxyl-terminal domain. It has a center domain comprising four proline-rich repeats that is responsible for the stimulation of actin-based motility; by binding to members of enabled/vasodilator-stimulated phosphoprotein (Ena/VASP) family it allows modulation of bacteria speed and directionality. An increased concentration of ActA at one pole forms the comet tail [27].

\section{LLO}

Although Listeriolysin O (LLO) has limited activity in cytosol (as mentioned above), recently its ability to damage the plasma membrane of host cells has been demonstrated. It results in redistribution and exposure of exofacial phosphatidylserine. Calcium influx triggers the membrane repair cascade that subsequently scissors the protrusion. This free bacteria-containing vacuole either binds to the TIM-4 receptor on the macrophage via the exposed PS or is engulfed by the neighboring cells through efferocytosis. The host membrane repair mechanism can be affected by several host factors to prevent LLO activity, but the exact mechanisms remain unclear [61].

\section{Cyclic di-GMP role in invasion}

Cyclic di-GMP or in short c-di-GMP is a common bacterial second messenger [62]. The c-di-GMP is synthesized from two GTP molecules by diguanylate cyclases (DGCs) and degraded by c-di-GMP-specific phosphodiesterases (PDEs) [63]. In L. monocytogenes, it is reported that elevated levels of c-di-GMP leads to weakening of human cell invasion because of decreased levels of internalin genes [62]. Elbakush and colleagues reported that increased levels of c-di-GMP were proven to inhibit listerial invasion of host cells. This inhibition is caused by decreased levels of cofactors of listerial CodY activities suppressing L. monocytogenes PrfA virulence [46].

\section{Regulator PrfA gene induce biofilm development}

The PrfA, a virulence regulator is a main factor for biofilm formation. This PrfA gene is present in all L. monocytogenes and associated with the pathogenesis of the infection [64]. Listeria monocytogenes is known to have the ability to persist for long periods on utensils and environments of food industries, thus leading to biofilm formation. Additionally, it becomes difficult to completely eradicate this bacterium upon biofilm formation because it can survive under extreme conditions and develop resistance to disinfectants [64]. The biofilm formation is an important phase for $L$. monocytogenes to grow and survive. The L. monocytogenes that develops biofilm are resistant to antimicrobials, thus making it more challenging to control these organisms as compared with planktonic bacteria $[33,65]$. The biofilm formation is a multistep process which includes an initial reversible and irreversible adherence, followed by the development of microcolonies and spreading onto the surface. The final maturation step allows the biofilm to form a complex, 3-dimensional structure. Its growth is affected by the type of surface and the availability of competitive microorganisms. Lemon and colleagues (2010) revealed that the PrfA virulence regulator in L. monocytogenes has a significant role on the extracellular biofilm formation. Usually, regulator PrfA gene is important to switch from extracellular flagellum propelled bacteria to intercellular. However, their experimental design showed that PrfA promotes biofilm production after transition from extracellular phase to surface-adhered biofilm former. This characteristic makes PrfA a universal regulator of $L$. monocytogenes and offers a likely regulatory connection between lifestyles of biofilm formations and the intracellular phase [19]. Travier and colleagues demonstrated that a major L. monocytogenes virulence determinant ActA, a PrfA-regulated gene product enabling actin polymerization. This promotes intracellular motility and cell-to-cell spread, an important phase of L. monocytogenes aggregation and biofilm formation [66]. Overall, the association of PrfA and biofilm formations has been well discussed and proven to promote biofilm development in many other studies [67].

\section{Agr system}

The Agr operon (accessory gene regulatory) is comprised of 4 genes: $\operatorname{agr} B, \operatorname{agrD}, \operatorname{agrC}$ and $\operatorname{agrA}[68]$. It is a two-component signal transduction system (2CS), consisting of a histidine kinase (HK) (intramembrane-bound sensor protein that phosphorylates upon activation by stimulus inside or at the surface of cytoplasmic membrane) and a response regulator (RR) (a cytoplasmic protein that is phosphorylated upon activation of $\mathrm{HK}$, and is involved in regulating the expression of regulon) [69]. In Agr system, AgrC is the HK and AgrA is the RR [68]. Transcription of agr genes especially agrA and $\operatorname{agr} D$, are important during the early stage of adhesion. However, 
the regulation of transcription of $\operatorname{agr} B$, $\operatorname{agr} C$ and $\operatorname{agr} D$ appear to be complex as they demonstrated a significant decrease in transcription levels after initial attachment. The agr operon is positively autoregulated since the mutation studies of $\operatorname{agrA}$ or $\operatorname{agrD}$ showed decreased transcription levels of $\operatorname{agr} B$, $\operatorname{agrC}$ and $\operatorname{agr} D$, though agrA transcription is not agr-dependent. agrA was suggested to be continuously expressed to provide basal level of regulator that is available to respond to the stimuli. High signals in the cell environment will induce the expression of $\operatorname{agrC}$, $\operatorname{agr} A$, $\operatorname{agrB}$ and $\operatorname{agr} D$, thus enabling the signals to be transferred to the neighbouring cells and prepare the cells to monitor the amplified signal [68]. These observations suggest that the Agr system is involved in the initial stage of biofilm formations.

\section{FlaA}

FlaA is a bacterial flagella major protein [70]. L. monocytogenes is motile and flagellated at below $30^{\circ} \mathrm{C}$. There are studies that revealed FlaA played an important role in biofilm formation [71]. This flagellum was found to be involved in the initial cell attachment phase by overcoming the van der Waals forces [72] and subsequent maturation of biofilm. Inactivation of this gene could lead to formation of less dense biofilm as compared to that of wild type. Some recent evidences showed that FlaA is associated with plasma membrane changes or biofilm development in relation to DegU [33].

\section{DegU system}

DegU (encoded by Imo2515) regulates motility, biofilm formation, and virulence in L. monocytogenes [73]. L. monocytogenes biofilm formation depends on the flagellar motility to propel the cells towards a surface prior to attachments [74]. The DegU system acts as an activator of flagellum biosynthesis [73]. At temperature below $30^{\circ} \mathrm{C}$, L. monocytogenes has four to six flagellar. These flagellum is glycosylated at multiple sites on flagelin protein FlaA [75]. But, L. monocytogenes becomes non-motile at temperature above $37^{\circ} \mathrm{C}$ by controlled repression of fla $\mathrm{A}$ transcription. The transcription takes place by by protein MogR that binds to the flaA upstream promoter region [38]. At low temperatures DegU activates transcription of $g m a R$, which encodes GmaR, the transcriptional activator of flaA $[33,73]$.

\section{D-alanylation pathway gene}

The D-alanylation pathway genes encoded by dItABCD was discovered to play a role in the biofilm formation of L. monocytogenes [65]. This recent study revealed that the $\operatorname{dltABCD}$ is involved in catalyzing the incorporation of D-alanine into lipoteichoic acids. This process, known as D-alanylation results in alteration of bacterial cell surface charges (reduction of negative charges on teichoic acid) or alternatively altered cell wall thickness or rigidity, leading to repulsion of cationic antimicrobial peptides (CAMPs) and reduction of attachment and biofilm development [65]. Alonso and colleagues suggested additional work is required to elucidate the specific role of this pathway gene in biofilm formation.

\section{Phosphate -sensing 2CS}

The phosphate-sensing two-component system (PhosphateSensing 2CS) phoPR operon expression is dependent on the concentration of inorganic phosphate. Since phosphate is essential for the synthesis of nucleotides and regulation of protein activity, under phosphate starvation conditions, bacteria will promote the synthesis of proteins using limited resources and make alternative sources of phosphorus. It was thus deduced that $\triangle p h o P R$ is unable to maintain the homeostasis of the cell envelope in relation to alteration of phosphate levels in the environment [65].

Biofilm is responsible for many post-processing contaminations of food. As described above, the complexity of each gene and their inter-relationship still requires further investigation. This knowledge will be of great importance as it may assist in development of eradication methods in the future.

\section{Clinical characteristics}

Although L. monocytogenes is an uncommon cause of illness in the general population, it can have severe clinical consequences that require hospitalization. It is the leading cause of death among major foodborne infections with a high mortality rate of $20-23 \%$. [76]. Infants, young children, elderly individuals, pregnant women, and patients with underlying diseases or immunocompromised conditions such as those on immunosuppressive medication or acquired immunodeficiency syndrome (AIDS) are vulnerable to listeriosis [77]. Furthermore, patients on antacids and H2-blocker medications which reduce gastric acidity are at risk as the oral infective dose of L. monocytogenes is reduced [42]. A listeriosis case is diagnosed based on isolation of L. monocytogenes from an ideally sterile site such as blood, cerebrospinal fluid, and products of conceptions.

The clinical manifestation of listeriosis ranges from mild febrile gastroenteritis to invasive disease, including bacteremia, encephalitis, meningitis and meningoencephalitis, rhomboencephalitis, or brain abscess. Listeriosis rarely manifests as focal illness, for example, pneumonia, cellulitis, lymphadenitis, conjunctivitis, and osteomyelitis.

In healthy individuals, listeriosis is generally accompanied by a mild febrile gastroenteritis, which is typically self-limiting, after ingestion of large amounts of bacteria (high infectious dose, estimated to be $10^{7}$ to $10^{8}$ colony-forming unit (CFU) $[29,76,78]$. According to an active surveillance program in the United States, sepsis is the most common illness in non-pregnant adults, followed by men- 
ingitis, and meningoencephalitis [77]. In the case of sepsis, most patients have symptoms of fever, fatigue, malaise and abdominal pain.

In the central nervous system (CNS), listeriosis infection targets the meninges and brain parenchyma especially the brain stem. Although the clinical manifestations of listeria meningitis are generally comparable to other bacterial meningitis, features such as tremors and grand mal and focal motor seizures are more commonly associated with listeria meningitis, suggesting more extensive CNS invasion [79]. Moreover, Listeria can cause brain stem encephalitis, brain abscess, and meningoencephalitis with multiple cranial nerve involvement, hemiparesis, ataxia, as well as respiratory arrest.

As an opportunistic pathogen, patients with the underlying disease may develop endocarditis involving native or prosthetic valves. Although it is rare, it can have devastating effects that subsequently lead to valve dysfunction and heart failure. However, compared to CNS infection, the mortality rate is slightly lower (about $12 \%$ ), possibly due to the extracellular location of the pathogen [80]. Listeriosis infection is 20 times more common in pregnant women compared with the general population [81]. The infection normally manifests with mild maternal illness - flu-like symptoms but then affects the fetus, with varying results at the different stages of conception potentially resulting in spontaneous abortion, preterm labor and neonatal infection such as pneumonia, sepsis and meningitis $[10,30,77,78]$. The risk of infection can be reduced by avoiding consumption of unpasteurized dairy products, cheeses, food from delicatessen counters, leftover and RTE foods $[30,82]$. Since listeriosis in pregnancy is catastrophic and difficult to diagnose, blood cultures have to be considered in febrile pregnant women, with influenza-like or gastrointestinal symptoms [30].

Listeriosis infection in a newborn can be divided into early-onset infection which results from infection in utero and late-onset infection due to exposure during birth or the period after that. Newborns with early-onset listeriosis have more severe clinical manifestations and there is a high chance of them being moribund as treatment cannot be administrated in time to affect the course of infection. Newborns with late-onset infection generally have a better prognosis with a cure rate of $80 \%$ - provided antibiotic regimen is given early. Neonatal meningitis often presents with temperature instability, lethargy, respiratory distress, poor feeding, vomiting and diarrhea; whereas stiff neck, bulging fontanelle, convulsions, and opisthotonus may not always be evident [80].

\section{Therapeutic regimens}

The treatment recommendations for listeriosis are generally based on the type, site and severity of infection as well as the im- mune status of the host. Patients developing gastroenteritis after consuming Listeria-contaminated food usually undergo a selflimiting course with symptom resolution within 2 days. Thus, immunocompetent patients rarely require antibiotics, although oral ampicillin or trimethoprim-sulfamethoxazole (TMP-SMX) may be administered to immunocompromised patients because they are at risk of developing an invasive listeriosis [83].

In patients with invasive Listeriosis, such as meningitis, bacteremia, and endocarditis, mortality remains high despite appropriate antibiotic treatment [80]. Due to the scarcity of listeriosis cases, prospective and randomized clinical studies on the most appropriate antibiotic regimes are lacking, making it difficult to identify the optimal antibiotic therapy for invasive infections $[30,84]$.

Although L. monocytogenes is susceptible in vitro to most of the commonly used antibiotics, treatment failure has been reported in $>30 \%$ cases [80]; this is caused by many factors. Since L. monocytogenes is an intracellular bacteria, it may evade the action of antibiotics as well as the host immune response [79]. Moreover, conventional in vitro susceptibility testing does not necessarily translate into clinical efficacy for intracellular bacteria. Only a few antibiotics such as cotrimoxazole, aminoglycosides, vancomycin, and the newer quinolones demonstrate bactericidal activity against Listeria, whereas beta-lactams are generally bacteriostatic [80]. Furthermore, the body's immune system may not be strong enough to help overcome the invading pathogen because listeriosis occurs mainly in immunocompromised hosts $[79,80]$.

The primary therapeutic options for treatment of severe listeriosis are ampicillin and penicillin [85]. Irrespective of central nervous system (CNS) involvement, a high dose of ampicillin is recommended because of the inclination of Listeria towards CNS infections [85], and to attain bactericidal concentration in the CNS [80]. Gentamicin, an aminoglycoside is often used in combination with penicillin and ampicillin to achieve synergy. Because of inherent resistance of $L$. monocytogenes to cephalosporins, this drug, which is commonly used as the first-line empirical treatment for meningitis, will not treat Listeria meningitis, depriving patients of effective treatment. Therefore, at-risk patients should be empirically treated for coverage of L. monocytogenes [80]. Antibiotics for treatment of meningitis should be administered for at least 21 days, with longer therapy ( $\geq 8$ weeks) in cases of rhombencephalitis, brain abscess, and immunocompromised host [85].

Bacterial TMP-SMX has high oral bioavailability and can be used as an alternative for patients allergic to penicillin. However, since TMP-SMX has anti-folate activity, it is best to avoid TMP-SMZ in pregnancy and in patients with risk of neural tube defects [30,82]. In the case of rifampicin, although it penetrates well into the host cell and demonstrates good intracellular activity, its use as mono- 
therapy is not recommended due to the reported resistance and limited clinical experience [82].

Moxifloxacin, a new generation of fluoroquinolone, is proposed to be a promising treatment alternative in the case of first line treatment failure or in severe cases where beta-lactams and TMPSMX are contraindicated. It has been shown to be effective in vitro against L. monocytogenes, this demonstrates its good intracellular penetration, ability to cross the blood-brain barrier, and diffuse into brain parenchyma. No relapse was reported in animals treated with repeated doses of moxifloxacin [84]. Carbapenems have good antimicrobial activity In vitro against $L$. monocytogenes with favorable intracellular activities. However, imipenem has the potential to cause seizures, therefore meropenem is recommended for the treatment of listeriosis, although failure with meropenem has been reported [80].

Since the recovery of listeriosis is dependent on the individuals' cell-mediated immunity, adjunctive therapy with immunomodulators, which are able to reconstitute the defective defense capacity, should theoretically help contain the infection. However, studies with L. monocytogenes - infected cells showed antagonistic activities against $\beta$-lactams antibiotics in interferon-treated cells, which would mean limited action of ampicillin against intracellular Listeria In vivo [86]. The use of corticosteroids suppresses the overwhelming inflammatory response in bacterial meningitis. However, since Listeria is an intracellular bacteria, macrophages and T-lymphocytes are the body's main defense mechanisms. The symptoms of patients with listeriosis are worsened if corticosteroids are administered as treatment [80].

\section{Conclusion}

Listeriosis is a rare but serious illness that affects all ages but is predominantly of concern in the immunocompromised population. The severity of this disease has led to a public warning about the importance of surveillance and prevention of Listeria. The US government has always maintained a "zero tolerance" policy of $L$ monocytogenes in all ready-to-eat food, however, other countries do not seem to take any precautionary actions to address this illness. It is yet unclear about the causes of the significant low prevalence of cases in the developing countries which might perhaps due to dietary habit or inefficient health surveillance system [87]. The increasing scenario of antibiotic resistance among bacteria in the environment has hindered the control and management of listeriosis. In view of the current situation, the US Food and Drug Administration (FDA) has approved the use of "lytic cocktail" - a form of bacteriophage in spray form to control the presence of $L$. monocyctogenes in food [88]. The efficacy of clinical antibiotics to treat bacterial infections have decline due to the extensive use of antibiotics in aquaculture and spread of multidrug resistance strains [89]. Aquaculture farmers can adapt the "switching meth- od" of antibiotics during the cultivation process in order to allow the withdrawal of antimicrobial resistance in bacteria [90]. In human infection, appropriate antibiotic regimens should be promptly instituted to patients. The community should always be educated about $L$. monocyctogenes and listeriosis by awareness campaigns and health screenings in hospitals.

\section{Conflict of Interest}

The authors declare that the research was conducted in the absence of any commercial or financial relationships that could be construed as a potential conflict of interest.

\section{Author Contributions}

$\mathrm{K}-\mathrm{YL}, \mathrm{VL}, \mathrm{AD}$ performed the literature search, critical data analysis as well as the manuscript writing. JW-FL provided the figures for the mansucripts. PP, H-LS, B-HG, SHW, N-SAM, L-HL and K-GC provided vital guidance, proofreading and technical support for the completion of the project. L-HL and K-GC founded this review project.

\section{Funding}

This work was financially supported by PVC Award Grant (Project No. PVC-ECR-2016), MOSTI ScienceFund Grant (Project No. 0602-10-SF0300), External Industry Grants from Biotek Abadi Sdn Bhd (vote no. GBA-808138 and GBA-808813) and BioMerge Sdn Bhd (vote no. GMRG2018-01) awarded to L-HL,University of Malaya for Research Grants (FRGS: FP022-2018A, and HIR Grant no. H-50001-A000027) awarded to K-GC.

\section{Bibliography}

1. (WHO) WHO. WHO Fact Sheets: Food Safety (2017).

2. Radoshevich L and Cossart P. "Listeria monocytogenes: towards a complete picture of its physiology and pathogenesis". Nature Reviews Microbiology 16 (2017): 32.

3. Gandhi M and Chikindas ML. "Listeria: a foodborne pathogen that knows how to survive". International Journal of Food Microbiology 113 (2007): 1-15.

4. Hamon M., et al. "Listeria monocytogenes: a multifaceted model”. Nature Reviews Microbiology 4 (2006): 423.

5. Buchanan RL., et al. "A review of Listeria monocytogenes: an update on outbreaks, virulence, dose-response, ecology, and risk assessments". Food Control 75 (2017): 1-13.

6. EFSA. "Listeria monocytogenes contamination of ready-to foods and the risk for human health in the EU". European Food Safety Authority Journal 16 (2018): 1-173.

7. EFSA. "The European Union summary reprot on trends and sources of zoonoses, zoonotic agents and food-borne outbreaks in 2013". European Food Safety Authority Journal 13 (2015): 165. 
8. Gaul LK., et al. "Hospital-acquired listeriosis outbreak caused by contaminated diced celery-Texas, 2010". Clinical Infectious Diseases 56 (2012): 20-26.

9. Pouillot R., et al. "Infectious dose of Listeria monocytogenes in outbreak linked to ice cream, United States, 2015". Emerging Infectious Diseases 22 (2016): 2113.

10. CDC. "Multistate outbreak of listeriosis associated with Jensen Farms cantaloupe - United States, August-September 2011". Morbidity and Mortality Weekly Report 60 (2011): 1357-1358.

11. CDC. 2015b. Sprouts and investigation of human listeriosis cases (final update).

12. CDC. 2015c. "Multistate outbreak of listeriosis linked to commercially produced, pre-packaged caramel apples made from bidart bros". Apples (final update).

13. Salama PJ., et al. "Learning from listeria: safer food for all". Lancet 391 (2018): 2305-2306.

14. Olanya OM., et al. "Cost estimation of listeriosis (Listeria monocytogenes) occurrence in South Africa in 2017 and its food safety implications". Food Control 102 (2019): 231-239.

15. (FAS) U-FS. "Global agriculture information network (GAIN)" Report (2018).

16. Schuppler M and Loessner MJ. "The opportunistic pathogen Listeria monocytogenes: pathogenicity and interaction with the mucosal immune system". International Journal of Inflammation (2010).

17. Letchumanan V., et al. "Bile Sensing: The activation of Vibrio parahaemolyticus virulence". Frontiers in Microbiology 8 (2017).

18. Dussurget O., et al. "Listeria monocytogenes bile salt hydrolase is a PrfA-regulated virulence factor involved in the intestinal and hepatic phases of listeriosis". Molecular Microbiology 45 (2002): 1095-1106.

19. Lemon KP., et al. "The virulence regulator PrfA promotes biofilm formation by Listeria monocytogenes". Journal of Bacteriology 192 (2010): 3969-3976.

20. Gray MJ., et al. "How the bacterial pathogen Listeria monocytogenes mediates the switch from environmental Dr. Jekyll to pathogenic Mr. Hyde". Infection and Immunity 74 (2006): 2505-2512.

21. Sabet C., et al. "The Listeria monocytogenes virulence factor InlJ is specifically expressed in vivo and behaves as an adhesin". Infection and Immunity 76 (2008): 1368-1378.

22. Sabet C., et al. "LPXTG protein InlJ, a newly identified internalin involved in Listeria monocytogenes virulence". Infection and Immunity 73 (2005): 6912-6922.
23. Drolia $\mathrm{R}$ and Bhunia AK. "Crossing the intestinal barrier via Listeria adhesion protein and internalin A". Trends in Microbiology (2019).

24. Becattini S., et al. "Commensal microbes provide first line defense against Listeria monocytogenes infection". Journal of Experimental Medicine 214 (2017): 1973-1989.

25. Archambaud C., et al. "Impact of lactobacilli on orally acquired listeriosis". Proceedings of the National Academy of Sciences 109 (2012): 16684-16689.

26. Sun A., et al. "Isolation of Listeria monocytogenes small-plaque mutants defective for intracellular growth and cell-to-cell spread". Infection and Immunity 58 (1990): 3770-3778.

27. Swaminathan B., et al. "Listeria monocytogenes. In Doyle MP, Beuchat (ed), Food microbiology: fundamentals and frontiers". Food Microbiology, Washington: ASM Press (2007): 457-491.

28. Kim $\mathrm{H} .$, et al. " $\sigma \mathrm{B}$ contributes to Listeria monocytogenes invasion by controlling expression of inlA and inlB". Microbiology 151 (2005): 3215

29. Xayarath B and Freitag NE. "PrfA and the Listeria monocytogenes switch from environmental bacterium to intracellular pathogen". In Vasil ML, Darwin, A. J. (ed), Regulation of Bacterial Virulence. American Society of Microbiology, Washington (2013): 363-385.

30. Janakiraman V. "Listeriosis in pregnancy: diagnosis, treatment, and prevention". Reviews in Obstetrics and Gynecology 1 (2008): 179.

31. Mujahid S., et al. "Protein level identification of the Listeria monocytogenes sigma $\mathrm{H}$, sigma $\mathrm{L}$, and sigma $\mathrm{C}$ regulons". BMC Microbiology 13 (2013): 156.

32. Ribeiro V., et al. "Contributions of $\sigma \mathrm{B}$ and PrfA to Listeria monocytogenes salt stress under food relevant conditions". International Journal of Food Microbiology 177 (2014): 98-108.

33. Kumar S., et al. "A study on the effects of some laboratoryderived genetic mutations on biofilm formation by Listeria monocytogenes". World Journal of Microbiology and Biotechnology 25 (2009): 527-531.

34. Shin J-H., et al. " $\sigma \mathrm{B}-$ dependent protein induction in Listeria monocytogenes during vancomycin stress". FEMS Microbiology Letters 308 (2010): 94-100.

35. Chaturongakul S., et al. "Transcriptomic and phenotypic analyses identify coregulated, overlapping regulons among PrfA, CtsR, $\mathrm{HrcA}$, and the alternative sigma factors $\sigma \mathrm{B}, \sigma \mathrm{C}, \sigma \mathrm{H}$, and $\sigma \mathrm{L}$ in Listeria monocytogenes". Applied and Environmental Microbiology 77 (2011): 187-200.

36. Grubaugh D., et al. "The Vir $\mathrm{AB} \mathrm{ABC}$ transporter is required for VirR regulation of Listeria monocytogenes virulence and resistance to nisin". Infection and Immunity 86 (2018): e00901-917. 
37. Christiansen JK., et al. "The RNA-binding protein Hfq of Listeria monocytogenes: role in stress tolerance and virulence". Journal of Bacteriology 186 (2004): 3355-3362.

38. Shen A and Higgins DE. "The MogR transcriptional repressor regulates nonhierarchal expression of flagellar motility genes and virulence in Listeria monocytogenes". PLoS Pathogens 2 (2006): e30.

39. Kamp HD and Higgins DE. "Transcriptional and post-transcriptional regulation of the GmaR antirepressor governs temperature-dependent control of flagellar motility in Listeria monocytogenes". Molecular Microbiology 74 (2009): 421-435.

40. Pinheiro J., et al. "MouR controls the expression of the Listeria monocytogenes Agr system and mediates virulence". Nucleic Acids Research 46 (2018): 9338-9352.

41. Mandin P., et al. "VirR, a response regulator critical for Listeria monocytogenes virulence". Molecular Microbiology 57 (2005): 1367-1380.

42. Barbuddhe SB and Chakraborty T. "Listeria as an enteroinvasive gastrointestinal pathogen Molecular Mechanisms of Bacterial Infection via the gut". Springer (2009): 173-195.

43. Osanai A., et al. "Fibronectin-binding protein, FbpA, is the adhesin responsible for pathogenesis of Listeria monocytogenes infection". Microbiology and Immunology 57 (2013): 253-262.

44. Tsai Y-HL., et al. "Listeria monocytogenes internalins are highly diverse and evolved by recombination and positive selection". Infection, Genetics and Evolution 6 (2006): 378-389.

45. Bonazzi M., et al. "Listeria monocytogenes internalin and Ecadherin: from bench to bedside". Cold Spring Harbor Perspectives in Biology 1 (2009): a003087.

46. Elbakush AM., et al. "CodY-mediated c-di-GMP-dependent inhibition of mammalian cell invasion in Listeria monocytogenes". Journal of Bacteriology 200 (2018): e00457-e00517.

47. Lecuit M., et al. "A single amino acid in E-cadherin responsible for host specificity towards the human pathogen Listeria monocytogenes". The EMBO Journal 18 (1999): 3956-3963.

48. Nikitas G., et al. "Transcytosis of Listeria monocytogenes across the intestinal barrier upon specific targeting of goblet cell accessible E-cadherin". Journal of Experimental Medicine 208 (2011): 2263-2277.

49. Hilliard A., et al. "Genomic characterization of Listeria monocytogenes isolates associated with clinical listeriosis and the food production environment in Ireland". Genes 9 (2018): 171.

50. Pentecost M., et al. "Listeria monocytogenes internalin B activates junctional endocytosis to accelerate intestinal invasion". PLoS Pathogens 6 (2010): e1000900.
51. Smith MA., et al. "Nonhuman primate model for Listeria monocytogenes-induced stillbirths". Infection and Immunity 71 (2003): 1574-1579.

52. Neves D., et al. "Structure of internalin InlK from the human pathogen Listeria monocytogenes". Journal of Molecular Biology 425 (2013): 4520-4529.

53. Lindén SK., et al. "Listeria monocytogenes internalins bind to the human intestinal mucin MUC2". Archives of Microbiology 190 (2008): 101-104.

54. Burkholder KM and Bhunia AK. "Listeria monocytogenes uses Listeria adhesion protein (LAP) to promote bacterial transepithelial translocation and induces expression of LAP receptor Hsp60". Infection and Immunity 78 (2010): 5062-5073.

55. Drolia R., et al. "Listeria adhesion protein induces intestinal epithelial barrier dysfunction for bacterial translocation". Cell host and Microbe 23 (2018): 470-484.

56. Jagadeesan B., et al. "LAP, an alcohol acetaldehyde dehydrogenase enzyme in Listeria, promotes bacterial adhesion to enterocyte-like Caco- 2 cells only in pathogenic species". Microbiology 156 (2010): 2782-2795.

57. Gasanov U., et al. "Identification of the insulin-like growth factor II receptor as a novel receptor for binding and invasion by Listeria monocytogenes". Infection and Immunity 74 (2006): 566-577.

58. Meyer-Morse N., et al. "Listeriolysin $\mathrm{O}$ is necessary and sufficient to induce autophagy during Listeria monocytogenes infection". PloS One 5 (2010): e8610.

59. Birmingham CL., et al. "Listeriolysin 0 allows Listeria monocytogenes replication in macrophage vacuoles". Nature 451 (2008): 350.

60. Poussin MA., et al. "The ability of Listeria monocytogenes PIPLC to facilitate escape from the macrophage phagosome is dependent on host PKC $\beta$ ". Microbial Pathogenesis 46 (2009): 1-5.

61. Czuczman MA., et al. "Listeria monocytogenes exploits efferocytosis to promote cell-to-cell spread". Nature 509 (2014): 230.

62. Chen L-H., et al. "Cyclic di-GMP-dependent signaling pathways in the pathogenic Firmicute Listeria monocytogenes". PLoS Pathogens 10 (2014): e1004301.

63. Lamprokostopoulou A., et al. "Cyclic di-GMP signalling controls virulence properties of Salmonella enterica serovar Typhimurium at the mucosal lining". Environmental Microbiology 12 (2010): 40-53.

64. Koo OK., et al. "Influence of Listeria innocua on the attachment of Listeria monocytogenes to stainless steel and aluminum surfaces". Food Control 39 (2014): 135-138. 
65. Alonso AN., et al. "Identification of Listeria monocytogenes determinants required for biofilm formation". PloS one 9 (2014): e113696.

66. Travier L., et al. "ActA promotes Listeria monocytogenes aggregation, intestinal colonization and carriage". PLoS Pathogens 9 (2013): e1003131.

67. Luo Q., et al. "PrfA led to reduced biofilm formation and contributed to altered gene expression patterns in biofilm-forming Listeria monocytogenes". Current Microbiology 67 (2013): 372-378.

68. Rieu A., et al. "Agr system of Listeria monocytogenes EGD-e: role in adherence and differential expression pattern". Applied and Environmental Microbiology 73 (2007): 6125-6133.

69. Collins B., et al. "Assessing the contributions of the LiaS histidine kinase to the innate resistance of Listeria monocytogenes to nisin, cephalosporins, and disinfectants". Applied and Environmental Microbiology 78 (2012): 2923-2929.

70. Desvaux M and Hébraud M. "The protein secretion systems in Listeria: inside out bacterial virulence". FEMS Microbiology Reviews 30 (2006): 774-805.

71. Chang Y., et al. "Identification of genes involved in Listeria monocytogenes biofilm formation by mariner-based transposon mutagenesis". Applied Microbiology and Biotechnology 93 (2012): 2051-2062.

72. Santos T., et al. "Listeria monocytogenes biofilm adaptation to different temperatures seen through shotgun proteomics". Frontiers in Nutrition 6 (2019): 89.

73. Williams T., et al. "Response regulator DegU of Listeria monocytogenes regulates the expression of flagella-specific genes". FEMS microbiology letters 252 (2005): 287-298.

74. Lemon KP., et al. "Flagellar motility is critical for Listeria monocytogenes biofilm formation". Journal of Bacteriology 189 (2007): 4418-4424.

75. Murray EJ., et al. "A pivotal role for the response regulator DegU in controlling multicellular behaviour". Microbiology 155 (2009): 1-8.

76. Graves LM and Catherine Wright Donnelly. "Microbiological aspects of the investigation that traced the 1998 outbreak of listeriosis in the United States to contaminated hot dogs and establishment of molecular subtyping-based surveillance for Listeria monocytogenes in the PulseNet network". Journal of Clinical Microbiology 43 (2005): 2350-2355.

77. Ryser E., et al. "Compendium of methods for the microbiological examination of foods". 4 (2001): 343-356.

78. Orsi RH., et al. "Listeria monocytogenes lineages: genomics, evolution, ecology, and phenotypic characteristics". International Journal of Medical Microbiology 301 (2011): 79-96.
79. Southwick FS and Purich DL. "Intracellular pathogenesis of listeriosis". New England Journal of Medicine 334 (1996): 770776.

80. Hof H. "An update on the medical management of listeriosis". Expert Opinion on Pharmacotherapy 5 (2004): 1727-1735.

81. Lamont RF., et al. "Listeriosis in human pregnancy: a systematic review". Journal of Perinatal Medicine 39 (2011): 227-236.

82. Shoham SMD and Bartlett JMD. "Listeria monocytogenes". The Johns Hopkins University (2018).

83. Ooi ST and Lorber B. "Gastroenteritis due to Listeria monocytogenes". Clinical Infectious Diseases 40 (2005): 1327-1332.

84. Grayo S., et al. "Rapid eradication of Listeria monocytogenes by moxifloxacin in a murine model of central nervous system listeriosis". Antimicrobial Agents and Chemotherapy 52 (2008): 3210-3215.

85. Lorber B. "Listeria monocytogenes". In Mandell GL, Bennett, J. E., Dolin, R (ed), Mandell, Douglas, and Bennett's Principles and Practice of Infectious Diseases $7^{\text {th }}$ ed, Philadelphia, PA: Churchill Livingstone (2010): 2707-2713.

86. Scorneaux B., et al. "Effect of recombinant human gamma interferon on intracellular activities of antibiotics against Listeria monocytogenes in the human macrophage cell line THP-1". Antimicrobial Agents and Chemotherapy 40 (1996): 1225-1230.

87. Letchumanan V., et al. "A review on the characteristics, taxanomy and prevalence of Listeria monocytogenes". Progress in Microbes and Molecular Biology 1 (2018).

88. Letchumanan V., et al. "Insights into bacteriophage application in controlling Vibrio species". Frontiers in Microbiology 7 (2016): 1114.

89. Letchumanan V., et al. "Genome sequence of Vibrio parahaemolyticus VP152 strain isolated from Penaeus indicus in Malaysia". Frontiers in Microbiology 7 (2016): 1410.

90. Letchumanan V., et al. "An insight of traditional plasmid curing in Vibrio species". Frontiers in Microbiology 6 (2015): 735.

\section{Assets from publication with us}

- Prompt Acknowledgement after receiving the article

- Thorough Double blinded peer review

- Rapid Publication

- Issue of Publication Certificate

- High visibility of your Published work

Website: https://www.actascientific.com/

Submit Article: https://www.actascientific.com/submission.php Email us: editor@actascientific.com

Contact us: +919182824667 